\title{
In-depth bioinformatic analysis of lung cancer-associated microRNA targets
}

\author{
HAIYANG ZHANG ${ }^{*}$, HAO YANG* ${ }^{*}$ RUI ZHANG, CHENYU ZHANG, JUNFENG ZHANG and DONGHAI LI \\ Jiangsu Engineering Research Center for microRNA Biology and Biotechnology, State Key Laboratory of \\ Pharmaceutical Biotechnology, School of Life Sciences, Nanjing University, Nanjing, Jiangsu 210093, P.R. China
}

Received July 16, 2013; Accepted September 9, 2013

DOI: $10.3892 /$ or.2013.2762

\begin{abstract}
Lung cancer (LC) is the leading cause of cancerrelated mortality worldwide. However, few studies of its specific mechanisms useful for diagnosis or treatment exist. microRNAs (miRNAs) present one mechanism through which genes with diverse functions on multiple pathways can be simultaneously regulated at the post-transcriptional level. However, LC-associated pathways targeted by LC-related miRNAs (LC-miRNAs) remain completely unknown. In the present study, we investigated $8 \mathrm{LC}$-miRNAs previously identified as regulators in three molecular subtypes of LC. The results showed that LC-miRNAs may post-transcriptionally function mainly through manipulating the expression of nucleic acid binding proteins and transcription factors, and target genes for the LC-miRNAs were most prominently predicted to function in regulation of transcription. Our analysis also highlighted the potential of these LC-miRNAs to regulate the cell differentiation, proliferation, endocytosis and migration signaling logically required to cause an LC cell mainly through five canonical pathways (PI3K-Akt signaling pathway, pathways in cancer, MAPK signaling pathway, HTLV-I infection and focal adhesion). These findings may form a useful basis for potential future development of novel LC therapeutic treatments.
\end{abstract}

\section{Introduction}

microRNAs (miRNAs) are a class of non-coding RNAs $\sim 22$ nucleotides in length that hybridize to mRNAs and cause either translation repression or mRNA cleavage $(1,2)$, and which were first discovered by Lee et al during an investigation of the gene lin-14 in Caenorhabditis elegans (C.elegans) development in the early 1990s (3). miRNAs are recognized as a distinct

Correspondence to: Dr Donghai Li, Jiangsu Engineering Research Center for microRNA Biology and Biotechnology, State Key Laboratory of Pharmaceutical Biotechnology, School of Life Sciences, Nanjing University, 22 Hankou Road, Nanjing, Jiangsu 210093, P.R. China E-mail: donghaili@nju.edu.cn

${ }^{*}$ Contributed equally

Key words: lung cancer, microRNA, EGFR, pathway class of biological post-transcriptional regulators involved in most biological processes including differentiation, apoptosis, proliferation, the immune response and development (4-10). miRNAs are found in animals, plants and fungi and are usually transcribed by RNA polymerase II (1). We recently demonstrated that exogenous plant MIR 168a cross-kingdomly targets mammalian LDLRAP1, suggesting that miRNAs could represent a novel group of universal modulators that mediate animal-plant interactions at the molecular level (11). Also, miRNAs play crucial roles in disease progression including cancer, and they may act as either tumor suppressors or oncogenes depending on the biological functions of their target genes (5). A wide variety of pathways are affected by miRNAs through regulating gene expression at the post-transcriptional level.

Lung cancer (LC) is the leading cause of cancer-related mortality worldwide and is commonly induced by long-term exposure to tobacco smoke (12). The present pathologic staging on the basis of morphology is inadequate to predict outcome for patient treatment. The emergence of molecular target therapy has improved the management of LC patients according to differentially expressed molecules in several subtypes. The identification and classification of LC-miRNArelated pathways for the analysis of several molecular subtypes of LC (KRAS, EGFR, ALK, BRAF, PIK3CA, MET, HER2, MEK1 and NRAS) raises the question of whether these approaches can be used to characterize and treat human LC. Identifying the molecular causes of LC represented a major breakthrough in the history of medicine, moving the discipline from pattern recognition and therapeutic strategies based on syndromic pathophysiology to molecular mechanism and evidence-based therapies derived from clinical trials designed on the basis of molecular mechanism (13). Recent therapeutic advances include the use of epidermal growth factor receptor tyrosine kinase inhibitors (EGFR-TKIs) including gefitinib and erlotinib (14). These drugs would be most effective with patient selection on the basis of target expression. miRNAs are thought to be grossly dysregulated in LCs and may also serve as oncogenes or tumor suppressors (15). Thus, miRNAs can be applied to sub-classify non-small cell LC (NSCLC) accounting for $80 \%$ of LC cases (16). For NSCLC, in particular, previous results have indicated that miRNA expression patterns could be proposed biomarkers used for diagnosis, prognosis and personalized therapy (17-19). 
Table I. Predictions of each LC-miRNA target.

\begin{tabular}{lllr}
\hline LC-miRNA & Molecular subtype of LC & Expression in LC & No. of predicted target genes \\
\hline let-7 family (let-7a-i) & KRAS & Downregulation & 372 \\
miR-7 & EGFR & Downregulation & 444 \\
miR-17 & MEK & Upregulation & 468 \\
miR-21 & EGFR & Upregulation & 164 \\
miR-96 & KRAS & Upregulation & 434 \\
miR-125a-5p & EGFR & Downregulation & 339 \\
miR-128b & EGFR & Downregulation & 1,039 \\
miR-145 & EGFR & Downregulation & 731 \\
\hline
\end{tabular}

In the present study, we summarized 8 LC-miRNAs which are associated with three subtypes of LC from previous studies. Protein class, molecular function, biological process and canonical pathways involved by the targets of each LC-miRNA as well as the 5 main canonical pathways all participated with certain LC-miRNAs, and were identified and analyzed, which may offer significant treatment insight into the clinical therapy of LC.

\section{Materials and methods}

miRNA target predictions. TargetScan (http://www.targetscan. org) was used to predict biological targets of miRNAs by searching for the presence of conserved $8 \mathrm{mer}$ and $7 \mathrm{mer}$ sites that match the seed region of each LC-miRNA (20) and generate lists of predicted gene targets of each miRNA. The list of targeted genes was input into PANTHER (Protein ANalysis THrough Evolutionary Relationships, http://www. pantherdb.org/) classification system designed to classify proteins in order to facilitate high-throughput analysis (21). The web-based functional annotation tool Database for Annotation, Visualization and Integrated Discovery (DAVID) v6.7(http://david.abcc.ncifcrf.gov/tools.jsp) has key components for disease analysis, Gene Ontology analysis and pathway analysis (22).

Signaling pathway mapping. The signaling pathways and processes were explored using the systems biology tool KEGG Mapper (http://www.genome.jp/kegg/tool/map_pathway2. html) which is a collection of tools for KEGG mapping: KEGG pathway mapping, BRITE mapping and MODULE mapping (23). The KEGG database consists of the 16 main databases (systems information, KEGG PATHWAY, KEGG BRITE, KEGG MODULE, KEGG DISEASE, KEGG DRUG and KEGG ENVIRON; genomic information, KEGG ORTHOLOGY, KEGG GENOME, KEGG GENES, KEGG SSDB and KEGG; chemical information, KEGG COMPOUND, KEGG GLYCAN, KEGG REACTION, KEGG RPAIR, KEGG RCLASS and KEGG ENZYME).

\section{Results and Discussion}

LC-associated miRNAs. Based on previous experimental data, 8 miRNAs were summarized as LC-miRNAs within three molecular subtypes (Table I). These LC-miRNA deregulations could drive tumorigenesis, through the roles LC-miRNAs can adopt as tumor suppressors or oncogenes in LC.

The let-7 family (let-7a to i), a conserved anti-oncomir set, is identified as a post-transcriptional gatekeeper during the cell proliferation process. The genomic regions located at let-7 family are usually eliminated in LC (24). Univariate analysis showed that downregulation of let-7 gene expression in NSCLC patients is correlated with poor prognosis $(25,26)$. Cell cycle arrest and cell death were caused by the expression of let-7g in K-Ras (G12D)-expressing murine LC cells and its overexpression clearly inhibited growth of both murine and human non-small cell lung tumors in tumor xenografts (27).

miR-7, a potential tumor suppressor in various types of human cancer, is frequently downregulated in LC. miR-7 overexpression not only inhibited NSCLC cell proliferation, but also caused cell apoptosis and decreased tumorigenicity (28). LC is under EGFR-mediated oncogenesis (29), and miR-7 regulates the EGFR pathway through attenuating the activation of Akt and ERK (30) and modulates EGFR oncogenic addiction (31).

From a large-scale miRNome analysis on LC, some miRNAs have been found with well characterized cancer association, which include miR-17 and miR-21 (32). Occasional amplification of the miR-17-92 cluster locus has been reported in human LC (33). miR-17, belonging to the miR-17-92 cluster, is known to act as oncogenes in multiple malignancies. Expression of miR-17 promotes cell proliferation, suppresses apoptosis of cancer cells and induces tumor angiogenesis (34).

Cancer and other diseases result in the upregulation of the miR-21 expression, which regulates many target genes associated with cellular survival, apoptosis and cell invasiveness. miR-21, a specific oncomir, is elevated under EGFR signaling stimulated conditions, especially in the context of EGFRactivating mutations, suggesting that miR-21 is related to lung carcinogenesis in never smokers (35). The miR-21 overexpression induced by diseases is reported to be a valuable approach for the new therapeutic strategies.

The high expression of miR-96 is found in tumor and serum, which may be considered a potential novel biomarker for the diagnosis and prognosis of $\mathrm{LC}$, and the increase of miR-96 expression was also related with the overall poor survival in patients with LC (36). Although evidence indicates that miR-96 decreased pancreatic cancer cell invasion and migration and slowed tumor growth in a manner associated with KRAS downregulation (37), miR-96 appears to be a potential oncomir in LC. 
A

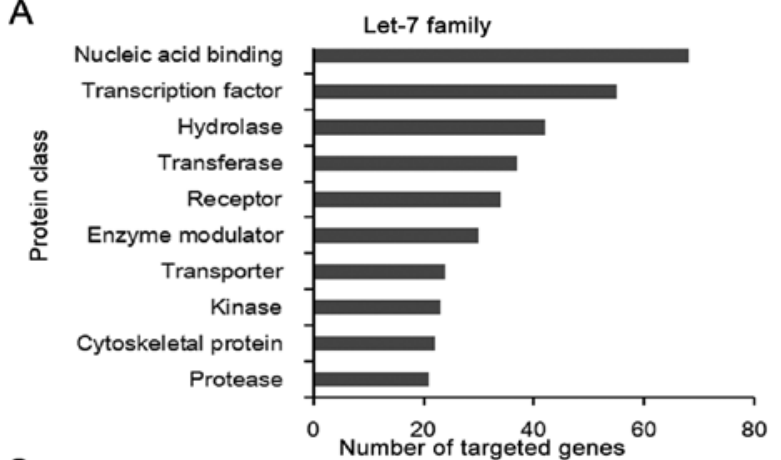

C

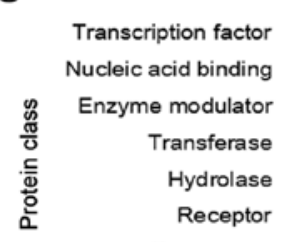

E

G

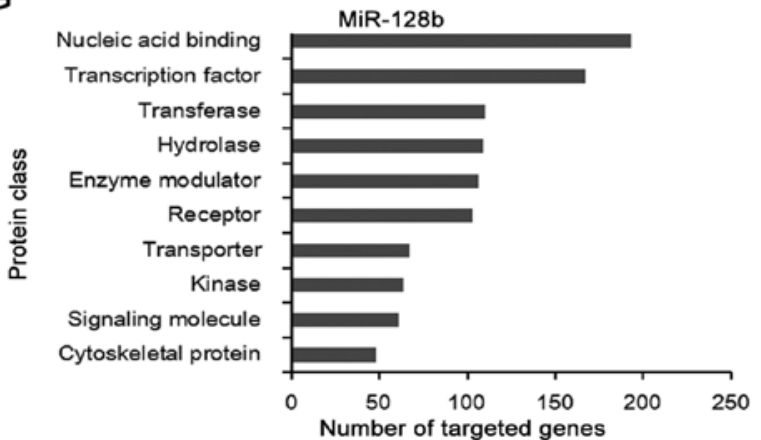

B
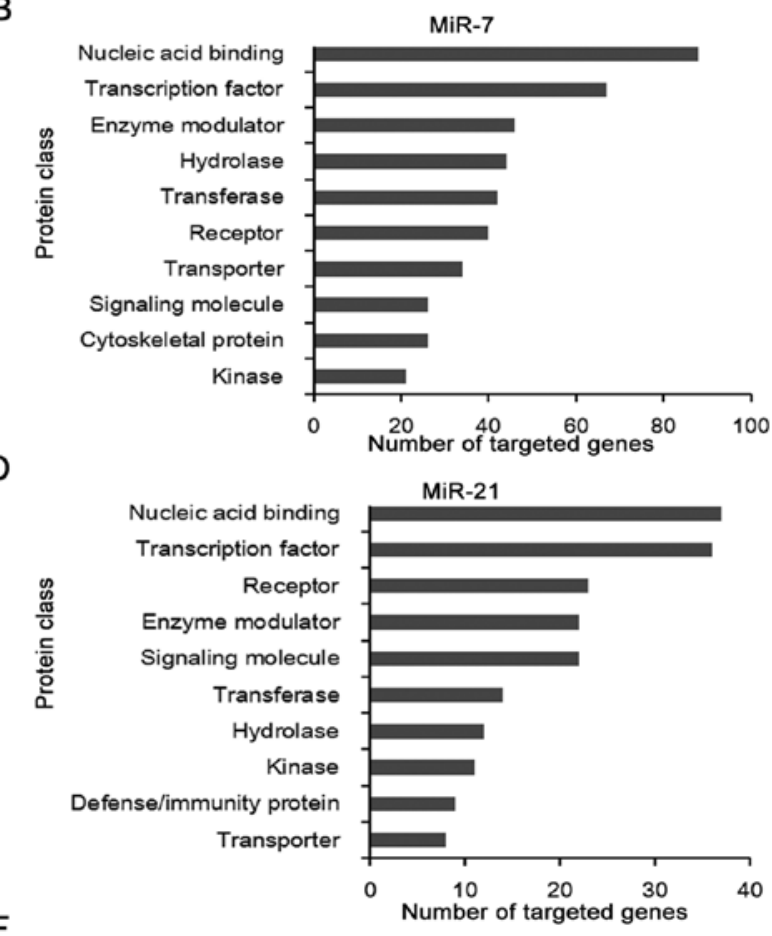

$\mathrm{F}$

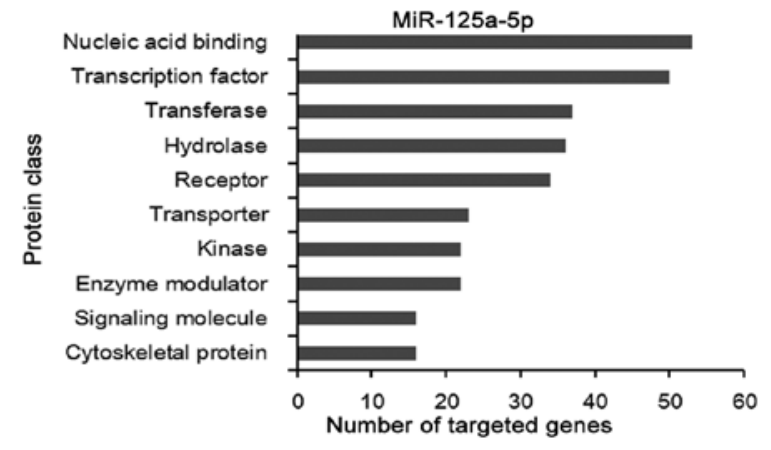

$\mathrm{H}$

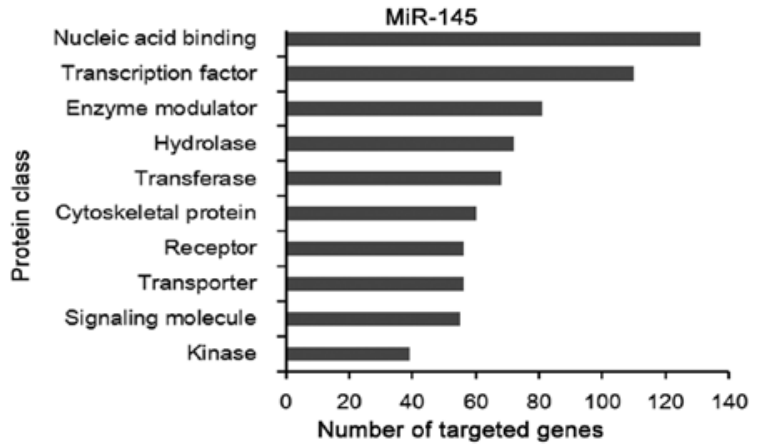

Figure 1. Major class of potential targets of LC-miRNAs.

The miR-125 family has been implicated in a variety of carcinomas as a tumor-suppressor. In LC, miR-125a-5p was reported to be stimulated by EGFR and served as a metastatic suppressor for the purpose of restraining tube formation and tumor formation (38). Jiang et al (39) stated that miR-125a-5p suppresses proliferation and induces apoptosis via a p53-dependent pathway in LC cells.

Admittedly, miRNA-128b is located on chromosome 3p and a putative regulator of EGFR and was proven to be correlated with response to targeted EGFR inhibition (40). Particularly, miR-128b loss-of-heterozygosity is continually identified in NSCLC patients and is closely associated with clinical response and survival after gefitinib treatment.

miR-145 is a tumor suppressor in the carcinogenesis of lung adenocarcinoma (41). miR-145 has been shown to suppress NSCLC cell proliferation by targeting c-Myc (42), lung adenocarcinoma-initiating cell proliferation by targeting OCT4 (43), cell proliferation of human lung adenocarcinoma by targeting EGFR and NUDT1 (44) and cell invasion and metastasis by directly targeting mucin 1 (45).

Thus, the let-7 family (let-7a to i), miR-7, miR-125a-5p, miR-128b and miR-145 are classified as anti-oncomirs 
Table II. Molecular function and biological process analysis of each LC-miRNA.

\begin{tabular}{|c|c|c|c|}
\hline LC-miRNA & Molecular function and biological process & $\%$ Regulated by LC-miRNAs & P-value \\
\hline \multirow[t]{5}{*}{ let-7 family } & Regulation of transcription & 20.6 & $2.80 \mathrm{E}-04$ \\
\hline & Transcription & 14.4 & $5.20 \mathrm{E}-02$ \\
\hline & Regulation of transcription, DNA-dependent & 13.7 & $7.90 \mathrm{E}-03$ \\
\hline & Regulation of RNA metabolic process & 13.7 & $1.20 \mathrm{E}-02$ \\
\hline & Phosphorus metabolic process & 10.2 & $9.40 \mathrm{E}-05$ \\
\hline \multirow[t]{5}{*}{$\operatorname{miR}-7$} & Transcription factor activity & 8.0 & $7.80 \mathrm{E}-03$ \\
\hline & Enzyme binding & 6.2 & $1.10 \mathrm{E}-05$ \\
\hline & Ion binding & 28.2 & $2.80 \mathrm{E}-02$ \\
\hline & Metal ion binding & 27.7 & $2.40 \mathrm{E}-02$ \\
\hline & Cation binding & 27.7 & $3.10 \mathrm{E}-02$ \\
\hline \multirow[t]{5}{*}{$\operatorname{miR}-17$} & Transition metal ion binding & 22.1 & $2.90 \mathrm{E}-04$ \\
\hline & Zinc ion binding & 18.2 & $1.60 \mathrm{E}-03$ \\
\hline & Ion binding & 31.6 & $8.60 \mathrm{E}-04$ \\
\hline & Metal ion binding & 31.4 & $4.20 \mathrm{E}-04$ \\
\hline & Cation binding & 31.4 & $6.50 \mathrm{E}-04$ \\
\hline \multirow[t]{5}{*}{$\operatorname{miR}-21$} & Transition metal ion binding & 18.8 & $2.80 \mathrm{E}-03$ \\
\hline & DNA binding & 17.3 & $3.50 \mathrm{E}-05$ \\
\hline & Ion binding & 29.4 & $1.00 \mathrm{E}-01$ \\
\hline & DNA binding & 19.0 & $4.00 \mathrm{E}-02$ \\
\hline & Transcription regulator activity & 17.8 & $3.00 \mathrm{E}-04$ \\
\hline \multirow[t]{5}{*}{$\operatorname{miR}-96$} & Transition metal ion binding & 19.8 & $4.20 \mathrm{E}-02$ \\
\hline & DNA binding & 18.2 & $6.40 \mathrm{E}-03$ \\
\hline & Nucleotide binding & 16.0 & $2.90 \mathrm{E}-02$ \\
\hline & Purine nucleotide binding & 13.7 & 4.70E-02 \\
\hline & Purine ribonucleotide binding & 12.7 & $8.30 \mathrm{E}-02$ \\
\hline \multirow[t]{5}{*}{$\operatorname{miR}-125 a-5 p$} & Transition metal ion binding & 20.1 & $1.10 \mathrm{E}-02$ \\
\hline & DNA binding & 18.0 & $3.30 \mathrm{E}-03$ \\
\hline & Cation binding & 31.3 & $5.10 \mathrm{E}-05$ \\
\hline & Ion binding & 31.3 & $9.80 \mathrm{E}-05$ \\
\hline & Metal ion binding & 30.7 & $1.00 \mathrm{E}-04$ \\
\hline \multirow[t]{5}{*}{$\operatorname{miR}-128 b$} & Ion binding & 28.2 & $2.40 \mathrm{E}-04$ \\
\hline & Metal ion binding & 27.9 & $9.50 \mathrm{E}-05$ \\
\hline & Cation binding & 27.9 & $2.00 \mathrm{E}-04$ \\
\hline & Transition metal ion binding & 18.8 & $2.80 \mathrm{E}-03$ \\
\hline & DNA binding & 17.3 & $3.50 \mathrm{E}-05$ \\
\hline \multirow[t]{5}{*}{$\operatorname{miR}-145$} & DNA binding & 16.4 & $2.40 \mathrm{E}-02$ \\
\hline & Zinc ion binding & 15.8 & $4.90 \mathrm{E}-02$ \\
\hline & Transcription regulator activity & 11.8 & $5.30 \mathrm{E}-03$ \\
\hline & Transcription factor activity & 8.0 & $7.80 \mathrm{E}-03$ \\
\hline & Enzyme binding & 6.2 & $1.10 \mathrm{E}-05$ \\
\hline
\end{tabular}

or tumor suppressors, while miR-17, miR-21 and miR-96 are oncomirs or OG (oncogenes). Identifying miRNAs as regulators of oncogenes or tumor suppressors could have farreaching implications for LC patients, including improving patient selection for targeted agents, development of novel therapeutics or early biomarkers of disease.

Predictions and protein classifications of LC-miRNA targets. Many target genes are regulated by a single miRNA. Therefore, to advance the understanding of the integrated functions of miRNAs, all its targets should be analyzed. As shown in Table I, each LC-miRNA or miRNA family has the ability to target between 164 and 1,039 mRNAs of predicted genes, and, in addition, multiple binding sites were observed in the 3'UTRs of the mRNAs for a unique miRNA. A total of 3,081 unique targeted genes, regulated by these 8 identified LC-miRNAs, have been found. Of all the targeted genes, 2,436 items are from targets of anti-oncomirs, thus, these genes are more likely upregulated in LC cells. Consequently, 994 downregulated targeted genes have been discovered from that of oncomiRs. Moreover, 13 genes (ALX4, CD69, CPEB3, DCUN1D3, DUSP8, FAM126B, FGD4, GAB1, NR2C2, 
Table III. Canonical pathway analysis of each LC-miRNA.

\begin{tabular}{|c|c|c|c|}
\hline LC-miRNA & Canonical pathways & $\%$ Regulated by LC-miRNAs & P-value \\
\hline \multirow[t]{4}{*}{ let-7 family } & Pathways in cancer & 4.2 & $9.00 \mathrm{E}-04$ \\
\hline & MAPK signaling pathway & 3.7 & 9.60E-04 \\
\hline & p53 signaling pathway & 2.0 & 4.30E-04 \\
\hline & mTOR signaling pathway & 1.2 & $2.10 \mathrm{E}-02$ \\
\hline \multirow[t]{6}{*}{$\operatorname{miR}-7$} & Pathways in cancer & 3.2 & $6.80 \mathrm{E}-02$ \\
\hline & Focal adhesion & 3.0 & 4.40E-03 \\
\hline & Endocytosis & 2.8 & $6.30 \mathrm{E}-03$ \\
\hline & Regulation of actin cytoskeleton & 2.5 & 4.30E-02 \\
\hline & Neurotrophin signaling pathway & 2.1 & $1.20 \mathrm{E}-02$ \\
\hline & Ubiquitin mediated proteolysis & 2.1 & $2.10 \mathrm{E}-02$ \\
\hline \multirow[t]{5}{*}{$\operatorname{miR}-17$} & Pathways in cancer & 2.8 & $1.60 \mathrm{E}-05$ \\
\hline & MAPK signaling pathway & 2.7 & $1.90 \mathrm{E}-08$ \\
\hline & Endocytosis & 2.2 & $8.00 \mathrm{E}-10$ \\
\hline & Regulation of actin cytoskeleton & 1.8 & $3.70 \mathrm{E}-04$ \\
\hline & Focal adhesion & 1.8 & $1.40 \mathrm{E}-04$ \\
\hline \multirow[t]{7}{*}{$\operatorname{miR}-21$} & MAPK signaling pathway & 6.7 & $1.20 \mathrm{E}-04$ \\
\hline & Pathways in cancer & 5.5 & $9.50 \mathrm{E}-03$ \\
\hline & Cytokine-cytokine receptor interaction & 4.9 & $9.50 \mathrm{E}-03$ \\
\hline & Jak-STAT signaling pathway & 4.3 & 2.80E-03 \\
\hline & Pancreatic cancer & 3.7 & 4.80E-04 \\
\hline & Chemokine signaling pathway & 3.7 & 2.80E-02 \\
\hline & Regulation of actin cytoskeleton & 3.7 & 4.70E-02 \\
\hline \multirow[t]{6}{*}{$\operatorname{miR}-96$} & Calcium signaling pathway & 2.6 & 4.20E-03 \\
\hline & Endocytosis & 2.6 & $5.70 \mathrm{E}-03$ \\
\hline & Focal adhesion & 2.1 & $6.60 \mathrm{E}-02$ \\
\hline & Gap junction & 1.9 & 2.80E-03 \\
\hline & GnRH signaling pathway & 1.7 & 1.80E-02 \\
\hline & Melanogenesis & 1.7 & $1.90 \mathrm{E}-02$ \\
\hline \multirow[t]{4}{*}{$\operatorname{miR}-125 \mathrm{a}$} & MAPK signaling pathway & 2.7 & $6.10 \mathrm{E}-02$ \\
\hline & Axon guidance & 1.8 & $5.40 \mathrm{E}-02$ \\
\hline & TGF- $\beta$ signaling pathway & 1.5 & 4.90E-02 \\
\hline & Biosynthesis of unsaturated fatty acids & 0.9 & 4.70E-02 \\
\hline \multirow[t]{5}{*}{$\operatorname{miR}-128 b$} & Pathways in cancer & 3.2 & $1.00 \mathrm{E}-03$ \\
\hline & MAPK signaling pathway & 2.9 & $6.80 \mathrm{E}-04$ \\
\hline & Focal adhesion & 2.1 & $7.10 \mathrm{E}-03$ \\
\hline & Neurotrophin signaling pathway & 1.9 & $1.30 \mathrm{E}-04$ \\
\hline & Regulation of actin cytoskeleton & 1.9 & $4.90 \mathrm{E}-02$ \\
\hline \multirow[t]{5}{*}{ miR-145 } & MAPK signaling pathway & 3.9 & $1.40 \mathrm{E}-05$ \\
\hline & Axon guidance & 3.1 & $5.80 \mathrm{E}-08$ \\
\hline & Endocytosis & 3.1 & $2.20 \mathrm{E}-05$ \\
\hline & Pathways in cancer & 3.1 & 3.30E-02 \\
\hline & Focal adhesion & 2.6 & $1.70 \mathrm{E}-03$ \\
\hline
\end{tabular}

SNTB2, SOCS6, STAT3 and ZNF217) were found in the targets of 2 oncomirs, and 421 genes were identified as targets of at least two anti-oncomiRs in LC in which 5 genes (HIPK2, $M B D 2, N F I B, S P 1$ and $S R G A P 2)$ seemed to be common targets of 4 anti-oncomirs. Those common target genes could be potential targets for drug discovery.

Fig. 1 shows the protein class of potential targets of each LC-miRNA. The largest number of genes is targeted by miR-128b, whereas miR-21 targets the minimum amount of genes. Their target genes of various LC-miRNAs with different seed region sequences belong to similar protein classes, including transcription factor, nucleic acid binding, enzyme modulator, kinase, cytoskeletal protein, transferase and hydrolase. In agreement with our previous studies, nucleic acid binding and transcription factor are the most important protein classes of targeted genes of LC-miRNAs $(5,46)$. Nucleic acid binding is described as interacting selectively and non-covalently with any nucleic acid, which consists 


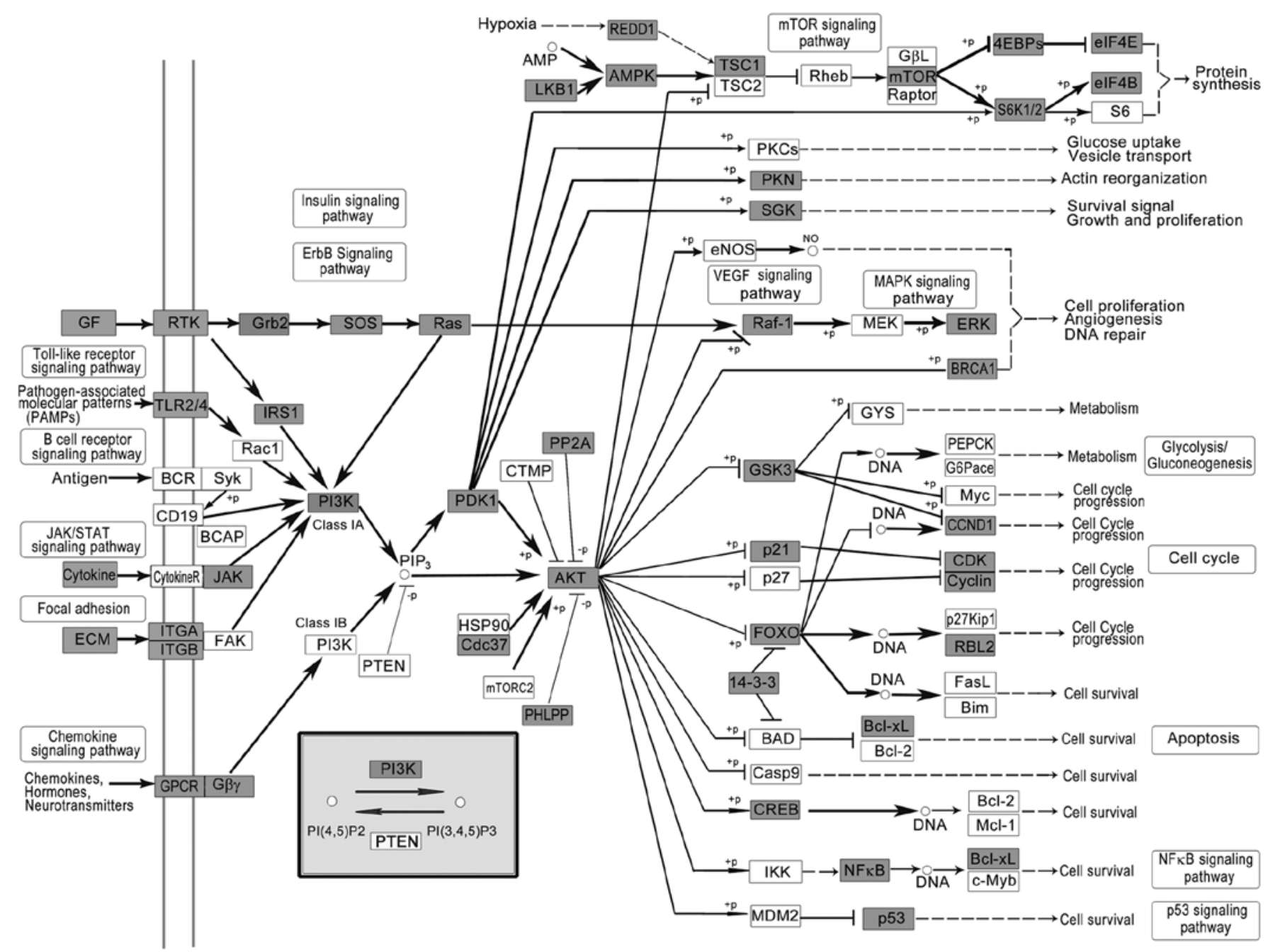

Figure 2. LC-miRNA regulation of PI3K-Akt signaling pathway biological map. Grey boxes show objects that can be regulated by LC-miRNAs. Solid arrows represent activation; dotted arrows indirect effect and $\perp$ inhibition. Letters on lines denote the type of regulation where $+\mathrm{p}$, phosphorylation; -p, dephosphorylation; +u, ubiquitination; +g, glycosylation; +m, methylation.

of base pairing, regulatory region nucleic acid binding, DNA binding, RNA binding, DNA/RNA hybrid binding and translation regulator activity with nucleic acid binding. These targets function as helicase, transcription factor, transmembrane receptor protein kinase, isomerase via nucleic acid binding during mRNA processing, DNA replication, cell cycle, tissue development and gamete generation. Of note, transcription factors control the activity of a gene by determining whether the gene's DNA is transcribed into mRNA and are detected in all living organisms. The number of transcription factors found within an organism increases with genome size, and larger genomes tend to have more transcription factors per gene (47). Transcription factors are vital for the normal development of an organism, as well as for routine cellular functions and response to disease. Recently, Kim et al (48) applied mass spectrometry to identify and quantify LC-related transcription factors by integrating previously reported genomic, transcriptomic, and proteomic data in various cell lines, and they found 14 differentially expressed transcription factors, such as STAT1 and SMAD4, which tuned genes associated with drug resistance and cell differentiation-related processes.
Molecular function, biological process and signaling pathway analysis of targets related to each $L C$-miRNA. To further clarify the functions of LC-miRNAs, molecular function, biological process and signaling pathway were analyzed and concluded. The top five items for these analyses of LC-miRNAs are listed in Tables II and III. Consistent with the aforementioned results in protein classification, over-represented molecular function and biological process of target genes are involved in transcription and binding, which are the dominant functions in the molecular regulation of mammals. Markedly, the targets for LC-miRNAs were most prominently predicted to function in pathways in cancer and MAPK signaling pathway shown in Table III, indicating that these miRNAs may regulate carcinogenesis mainly through the very two pathways. Due to the relevance of LC and multiple signaling pathway, endocytosis, regulation of actin cytoskeleton, p53 signaling pathway, mTOR signaling pathway and focal adhesion were also confirmed in $\mathrm{LC}$ as previously reported (5).

Pathway mapping of LC-miRNA targets. To sum up the pathway implicated in all targets of 8 LC-miRNAs, 3,081 unique targets were used for further pathway analysis. As 
Table IV. Top five pathways regulated by all 8 LC-miRNAs.

\begin{tabular}{lllll}
\hline Pathway DB & \multicolumn{1}{c}{ Name } & Hits & Total & Percent (\%) \\
\hline \multirow{2}{*}{ KEGG } & PI3K-Akt signaling pathway - Homo sapiens & 94 & 336 & 27.97 \\
& Pathways in cancer - Homo sapiens & 91 & 343 & 26.53 \\
& MAPK signaling pathway - Homo sapiens & 87 & 284 & 198 \\
& HTLV-I infection- Homo sapiens & 68 & 207 & 34.08 \\
& Focal adhesion - Homo sapiens & 64 & 30.92 \\
\hline
\end{tabular}

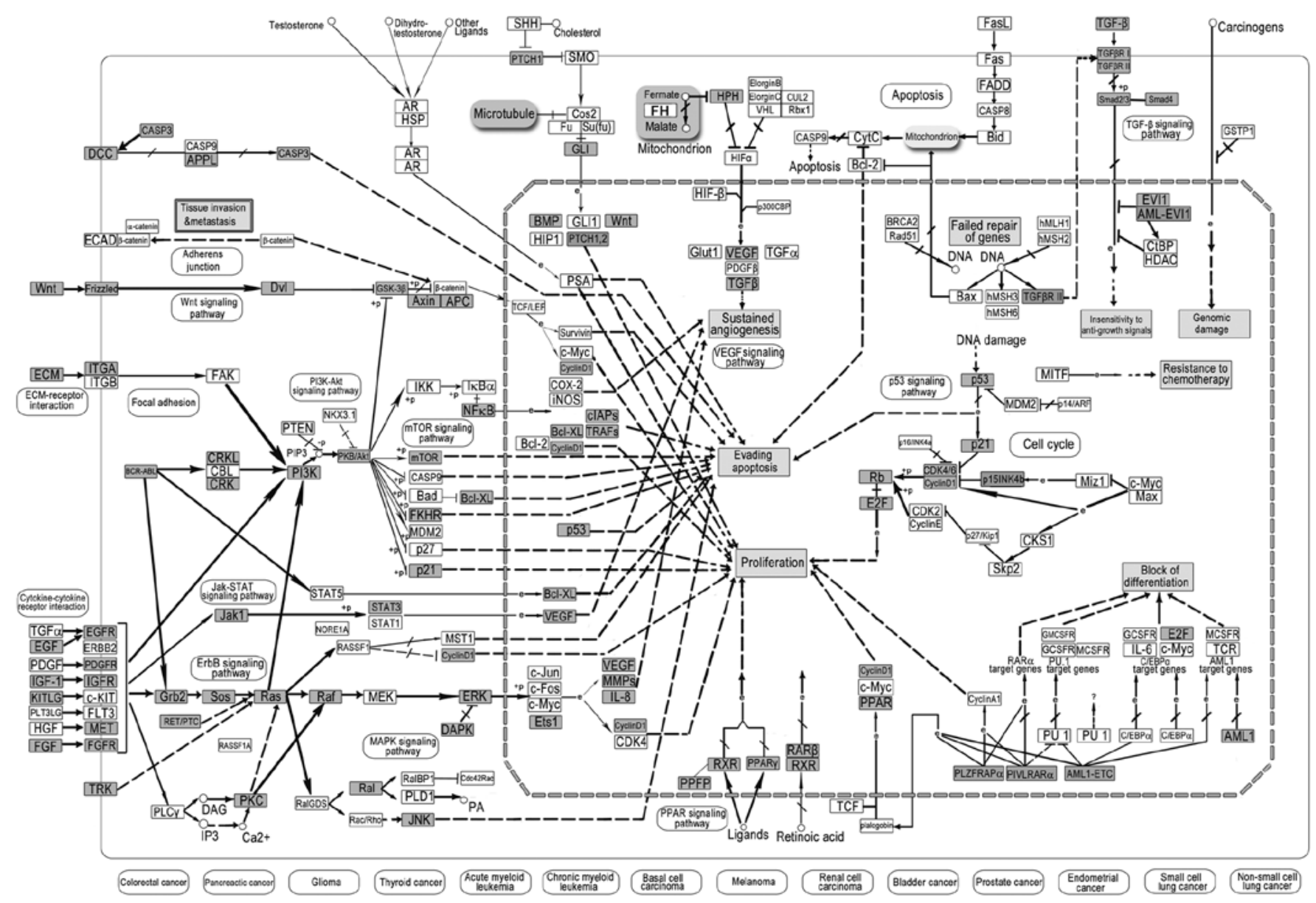

Figure 3. LC-miRNA regulation of pathways in cancer biological map. Grey boxes show objects that can be regulated by LC-miRNAs. Solid arrows represent activation; dotted arrows indirect effect and $\perp$ inhibition. Letters on lines denote the type of regulation where $+p$, phosphorylation; - $p$, dephosphorylation; $+\mathrm{u}$, ubiquitination; $+\mathrm{g}$, glycosylation; $+\mathrm{m}$, methylation.

shown in Table IV, PI3K-Akt signaling pathway, pathways in cancer, MAPK signaling pathway, HTLV-I infection and focal adhesion were the top five pathways regulated by 8 LC-miRNAs. These pathways have been demonstrated to play essential roles in morphological changes, intercellular communication and invasion of cancer.

Phosphatidylinositol-3-kinase (PI3K)-Akt signaling pathway (Fig. 2), a pathway thought to be specific to EGFR/ERBB family receptors, has been reported to change frequently in diverse human cancer (49). PI3K phosphorylates 3 position of the inositol ring of $\mathrm{PI}(4,5) \mathrm{P} 2$, to generate PI $(3,4,5)$ P3 (50). Previous studies have demonstrated that numerous components of the PI3K-AKT pathway rather than any other pathway in human cancer, crucial to many aspects of cell growth and survival, are altered by amplification, mutation and translocation frequently, with resultant activation of the pathway. Also, the PI3K/AKT pathway is targeted for LC drug discovery (51).

Pathway in cancer is a comprehensive biological signaling pathway related to tissue invasion and metastasis, genomic damage, insensitivity to anti-growth signals, resistance to chemotherapy, evading apoptosis, sustained angiogenesis, proliferation and block of differentiation, which also links to diversified cancers, including colorectal, pancreatic, thyroid, bladder, prostate, endometrial and LC. Many targets of LC-miRNAs were found in pathways in cancer (Fig. 3), 


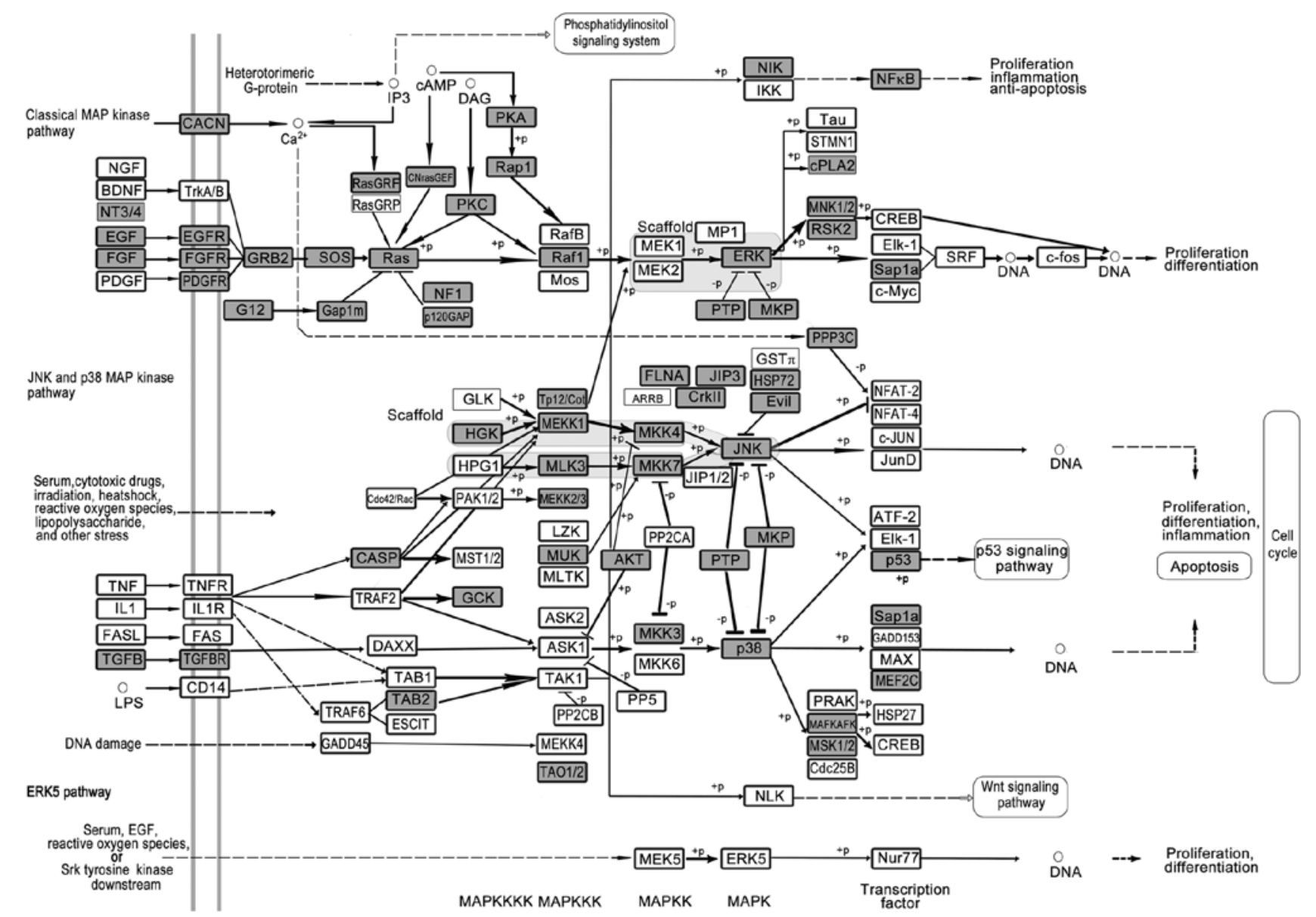

Figure 4. LC-miRNA regulation of MAPK signaling pathway biological map. Grey boxes show objects that can be regulated by LC-miRNAs. Solid arrows represent activation; dotted arrows indirect effect and $\perp$ inhibition. Letters on lines denote the type of regulation where $+\mathrm{p}$, phosphorylation; -p, dephosphorylation; +u, ubiquitination; +g, glycosylation; +m, methylation.

and these significant findings in LC may lead to customized therapy based on targeting specific genes. The signaling pathway could offer valuable roadmaps for drug discovery and therapy.

Mitogen-activated protein kinase (MAPK) cascade activated by peptide growth factors, cytokines, hormones, and various cellular stressors is a highly conserved module involved in various cellular functions, including cell proliferation, differentiation and migration (Fig. 4). Mammals express at least three distinctly regulated groups of MAPKs, extracellular signal-regulated kinase (ERK), p38 and c-Jun $\mathrm{NH} 2$-terminal kinase and each of these enzymes exists in several isoforms. The pathway has been suggested to function in several steps of tumorigenesis including cancer cell proliferation, migration and invasion. Lung metastasis was found to be substantially delayed in MEKK1 knockout mice (52). Mutations in EGFR activating the pathway occur frequently in LC (53). Experimental mouse models help to elucidate the mechanism of how these MAPKs control cancer development, and appear to provide new strategies for the design of improved therapeutic approaches (54). Small-molecule inhibitors designed to target various steps of this pathway have entered clinical trials (55).

Human T-lymphotropic virus type 1 (HTLV-1) is a pathogenic retrovirus associated with adult T-cell leukemia/lymphoma. Tax encoded by HTLV-I has been implicated in oncogenesis, which is a transcriptional co-factor that disturbs anti-apoptosis or cell proliferation (56). Although the infection rate of HTLV-1 may not be associated with increased risk of cancer, the Wnt, TGF $\beta$, Ras, NF- $\kappa B$, p53 in HTLV-1 infection pathways may play pivotal roles in lung carcinogenesis (Fig. 5).

Focal adhesion (Fig. 6), directly connected with cell mobility, proliferation and survival, was altered in LC, possibly due to the development of malignant LC, which is associated with perturbations in these processes. Inducible expression of an inhibitory focal adhesion kinase (FAK) protein and FAK-related non-kinase (FRNK) suppressed the growth of primary tumors and blocked metastasis formation in the lungs. Lung metastasis formation was nearly completely avoided when FAK-related non-kinase (FRNK) was already expressed prior to tumor cell injection; nevertheless, FRNK expression after injection did not influence lung metastasis formation (57).

We found specific pathways from the Top 10 pathways of each subtype of $\mathrm{LC}$ (Table V). The regulation of actin cytoskeleton pathway is specific to EGFR sub-type LC (EGFR-LC); cholinergic synapse and calcium signaling pathway belong to KRAS-LC; ubiquitin mediated proteolysis and cytokine-cytokine receptor interaction are unique to MEK-LC. Regulation of actin cytoskeleton pathway is often stimulated in invasive and metastatic LC cells as malignant LC cells utilize their intrinsic migratory ability to invade adjacent tissues and the 


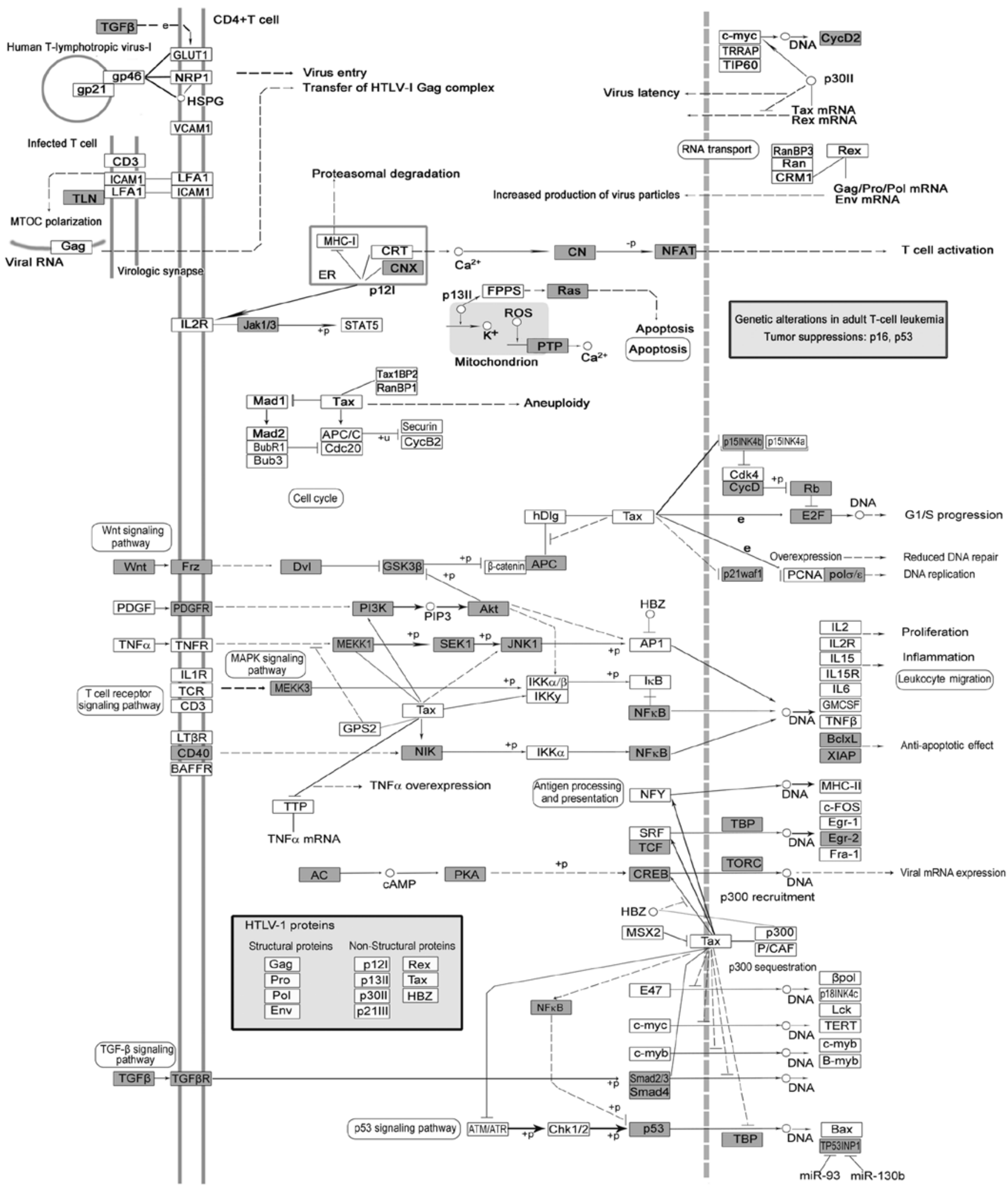

Figure 5. LC-miRNA regulation of HTLV-1 infection biological map. Grey boxes show objects that can be regulated by LC-miRNAs. Solid arrows represent activation; dotted arrows indirect effect and $\perp$ inhibition. Letters on lines denote the type of regulation where $+p$, phosphorylation; -p, dephosphorylation; $+\mathrm{u}$, ubiquitination; $+\mathrm{g}$, glycosylation; $+\mathrm{m}$, methylation.

vasculature and ultimately to metastasize (58). In particular, a subunit of Arp 2/3 complex is observed in malignant human LC and correlated with poor patient outcome which is in accordance with our results (59). Cholinergic synapse and calcium signaling pathways were closely related to LC since both muscarinic cholinergic receptor $3(\mathrm{mAChR} 3)$ and the nicotinic cholinergic receptor (nAChR) are expressed in small cell LC (SCLC). A number of LC studies have focused on 
Table V. Specific pathways of each subtype of LC with KEGG mapper.

\begin{tabular}{llrr}
\hline Subtype of LC & \multicolumn{1}{c}{ Specific pathways } & Hits & Percent $(\%)$ \\
\hline EGFR & Regulation of actin cytoskeleton - Homo sapiens & 50 & 23 \\
KRAS & Cholinergic synapse - Homo sapiens & 13 & 32 \\
KRAS & Calcium signaling pathway - Homo sapiens & 14 & 8 \\
MEK & Ubiquitin mediated proteolysis - Homo sapiens & 9 & 6 \\
MEK & Cytokine-cytokine receptor interaction - Homo sapiens & 9 & 3 \\
\hline
\end{tabular}

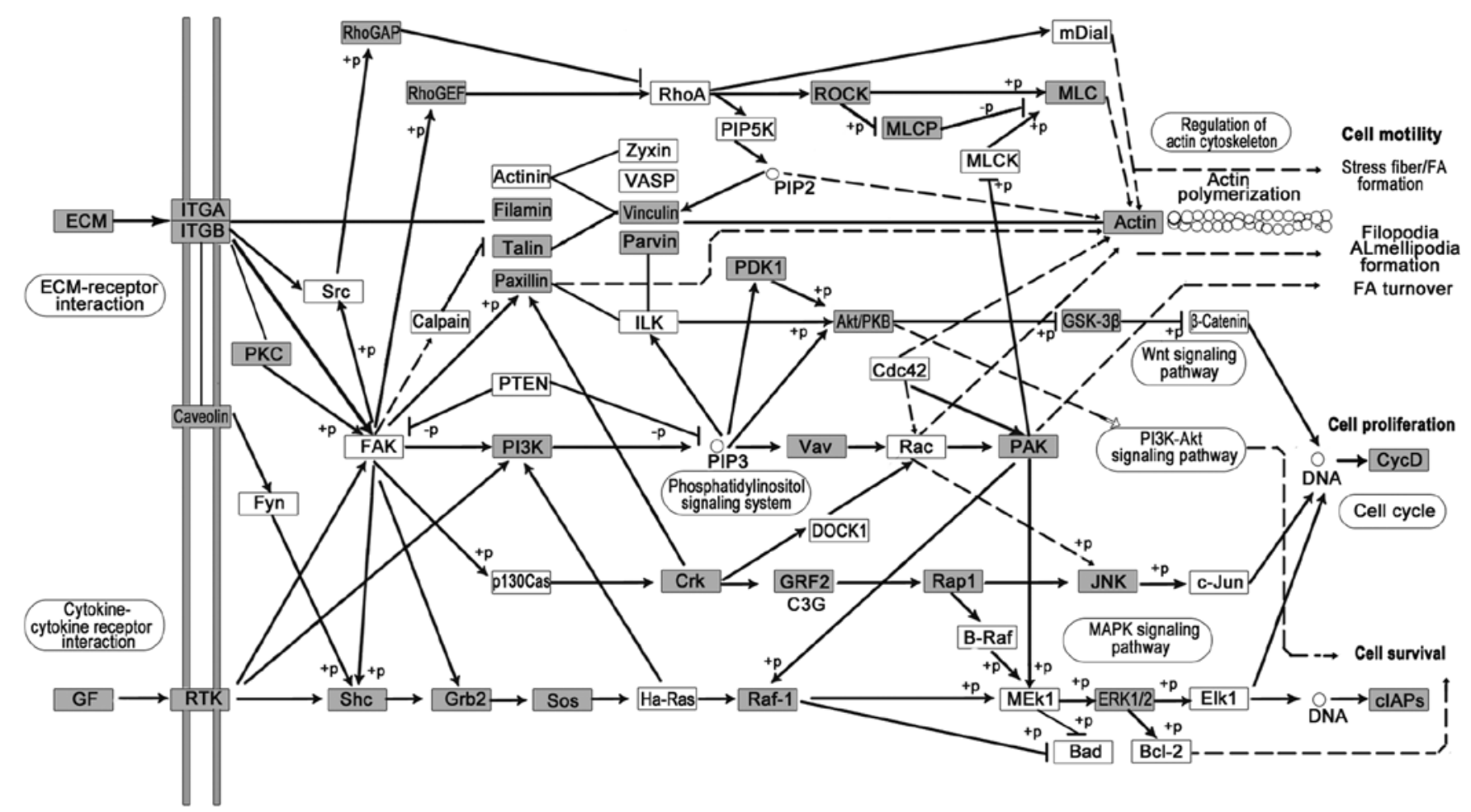

Figure 6. LC-miRNA regulation of focal adhesion biological map. Grey boxes show objects that can be regulated by LC-miRNAs. Solid arrows represent activation; dotted arrows indirect effect and $\perp$ inhibition. Letters on lines denote the type of regulation where $+p$, phosphorylation; -p, dephosphorylation; $+\mathrm{u}$, ubiquitination; $+\mathrm{g}$, glycosylation; $+\mathrm{m}$, methylation.

nAChR due to the close relationship between tobacco and LC. Specifically, nicotine stimulates LC carcinogenesis, proliferation and angiogenesis and also inhibits LC apoptosis induced by chemotherapeutic drugs through the nAChR (60). It is reported that the $\mathrm{mAChR} 3$ antagonist could be developed as a beneficial therapeutic approach for SCLC patients, notably those with a comorbidity of chronic obstructive pulmonary disease (61). Ubiquitin-mediated proteolysis can maintain protein homeostasis and is critical in regulating cancer-related cellular processes, and inhibitors of the proteasome and their molecular mechanisms for targeting substrate-specific E3 ligases that are likely to yield a new class of therapeutics that will serve as anti-LC drugs due to multitude of E3s and their specific substrate recognition (62). Cytokine-cytokine receptor interaction is important in cell-cell communications, whereas abnormal expression of cytokines or cytokine receptors could certainly contribute to LC.
In the present study, we used theoretical gene target identification and pathway mapping to create a biological framework by which to test the relevance of LC-miRNAs in LC induction. The recognition of LC-miRNA-related pathways, with the ability to regulate a complex pathological process in three molecular subtypes of LC, can be improved using bioinformatic techniques followed by experimental validation.

\section{Acknowledgements}

The present study was supported by grants from the National Natural Science Foundation of China (31000323, 31070672, 81250044), the Natural Science Foundation of Jiangsu Province (BK20131272), the Specialized Research Fund for the Doctoral Program of Higher Education of China (20100091120023) and the Fundamental Research Funds for the Central Universities (1095020823). 


\section{References}

1. Bartel DP: MicroRNAs: genomics, biogenesis, mechanism, and function. Cell 116: 281-297, 2004.

2. He L and Hannon GJ: MicroRNAs: small RNAs with a big role in gene regulation. Nat Rev Genet 5: 522-531, 2004.

3. Lee RC, Feinbaum RL and Ambros V: The C. elegans heterochronic gene lin-4 encodes small RNAs with antisense complementarity to lin-14. Cell 75: 843-854, 1993.

4. Lim LP, Lau NC, Garrett-Engele P, et al: Microarray analysis shows that some microRNAs downregulate large numbers of target mRNAs. Nature 433: 769-773, 2005.

5. Yang H, Zhang H, Zhu L, Wang J, Zhang C and Li D: Pathway analysis of cancer-associated microRNA targets. Int J Oncol 41 2213-2226, 2012

6. Zhu D, Pan C, Li L, et al: MicroRNA-17/20a/106a modulate macrophage inflammatory responses through targeting signalregulatory protein alpha.J Allergy Clin Immunol 132: 426-436.e8 2013.

7. Brennecke J, Hipfner DR, Stark A, Russell RB and Cohen SM: bantam encodes a developmentally regulated microRNA that controls cell proliferation and regulates the proapoptotic gene hid in Drosophila. Cell 113: 25-36, 2003.

8. Cuellar TL and McManus MT: MicroRNAs and endocrine biology. J Endocrinol 187: 327-332, 2005.

9. Poy MN, Eliasson L, Krutzfeldt J, et al: A pancreatic islet-specific microRNA regulates insulin secretion. Nature 432: 226-230, 2004

10. Chen CZ, Li L, Lodish HF and Bartel DP: MicroRNAs modulate hematopoietic lineage differentiation. Science 303: 83-86, 2004.

11. Zhang L, Hou D, Chen X, et al: Exogenous plant MIR168a specifically targets mammalian LDLRAP1: evidence of crosskingdom regulation by microRNA. Cell Res 22: 107-126, 2012.

12. Jemal A, Siegel R, Xu J and Ward E: Cancer statistics, 2010. CA Cancer J Clin 60: 277-300, 2010.

13. Loscalzo J, Kohane I and Barabasi AL: Human disease classification in the postgenomic era: a complex systems approach to human pathobiology. Mol Syst Biol 3: 124, 2007.

14. Nie W, Tang L, Zhang H, et al: Structural analysis of the EGFR TK domain and potential implications for EGFR targeted therapy. Int J Oncol 40: 1763-1769, 2012.

15. Croce CM: Causes and consequences of microRNA dysregulation in cancer. Nat Rev Genet 10: 704-714, 2009.

16. Bishop JA, Benjamin H, Cholakh H, Chajut A, Clark DP and Westra WH: Accurate classification of non-small cell lung carcinoma using a novel microRNA-based approach. Clin Cancer Res 16: 610-619, 2010.

17. Patnaik SK, Kannisto E, Knudsen S and Yendamuri S: Evaluation of microRNA expression profiles that may predict recurrence of localized stage I non-small cell lung cancer after surgical resection. Cancer Res 70: 36-45, 2010

18. Raponi M, Dossey L, Jatkoe T, et al: MicroRNA classifiers for predicting prognosis of squamous cell lung cancer. Cancer Res 69: 5776-5783, 2009.

19. Lin PY, Yu SL and Yang PC: MicroRNA in lung cancer. Br J Cancer 103: 1144-1148, 2010.

20. Lewis BP, Burge CB and Bartel DP: Conserved seed pairing, often flanked by adenosines, indicates that thousands of human genes are microRNA targets. Cell 120: 15-20, 2005.

21. Mi H, Muruganujan A and Thomas PD: PANTHER in 2013 modeling the evolution of gene function, and other gene attributes, in the context of phylogenetic trees. Nucleic Acids Res 41 D377-D386, 2013.

22. Huang da W, Sherman BT, Tan Q, et al: The DAVID Gene Functional Classification Tool: a novel biological module-centric algorithm to functionally analyze large gene lists. Genome Biol 8: R183, 2007.

23. Kanehisa M, Araki M, Goto S, et al: KEGG for linking genomes to life and the environment. Nucleic Acids Res 36: D480-D484 2008.

24. Calin GA, Sevignani C, Dumitru CD, et al: Human microRNA genes are frequently located at fragile sites and genomic regions involved in cancers. Proc Natl Acad Sci USA 101: 2999-3004, 2004.

25. Takamizawa J, Konishi H, Yanagisawa K, et al: Reduced expression of the let-7 microRNAs in human lung cancers in association with shortened postoperative survival. Cancer Res 64: 3753-3756, 2004.

26. Yanaihara N, Caplen N, Bowman E, et al: Unique microRNA molecular profiles in lung cancer diagnosis and prognosis. Cancer Cell 9: 189-198, 2006.
27. Kumar MS, Erkeland SJ, Pester RE, et al: Suppression of non-small cell lung tumor development by the let-7 microRNA family. Proc Natl Acad Sci USA 105: 3903-3908, 2008.

28. Xiong S, Zheng Y, Jiang P, Liu R, Liu X and Chu Y: MicroRNA-7 inhibits the growth of human non-small cell lung cancer A549 cells through targeting BCL-2. Int J Biol Sci 7: 805-814, 2011.

29. Wong KK: Searching for a magic bullet in NSCLC: the role of epidermal growth factor receptor mutations and tyrosine kinase inhibitors. Lung Cancer 60 (Suppl 2): S10-S18, 2008.

30. Webster RJ, Giles KM, Price KJ, Zhang PM, Mattick JS and Leedman PJ: Regulation of epidermal growth factor receptor signaling in human cancer cells by microRNA-7. J Biol Chem 284: 5731-5741, 2009.

31. Chou YT, Lin HH, Lien YC, et al: EGFR promotes lung tumorigenesis by activating miR-7 through a Ras/ERK/Myc pathway that targets the Ets2 transcriptional repressor ERF. Cancer Res 70: 8822-8831, 2010

32. Volinia S, Calin GA, Liu CG, et al: A microRNA expression signature of human solid tumors defines cancer gene targets. Proc Natl Acad Sci USA 103: 2257-2261, 2006.

33. Hayashita Y,Osada H,Tatematsu Y, et al: A polycistronic microRNA cluster, miR-17-92, is overexpressed in human lung cancers and enhances cell proliferation. Cancer Res 65: 9628-9632, 2005.

34. Mendell JT: miRiad roles for the miR-17-92 cluster in development and disease. Cell 133: 217-222, 2008.

35. Seike M, Goto A, Okano T, et al: MiR-21 is an EGFR-regulated anti-apoptotic factor in lung cancer in never-smokers. Proc Natl Acad Sci USA 106: 12085-12090, 2009.

36. Zhu W,Liu X,He J,Chen D, Hunag Y and Zhang YK: Overexpression of members of the microRNA-183 family is a risk factor for lung cancer: a case control study. BMC Cancer 11: 393, 2011.

37. Yu S, Lu Z, Liu C, et al: miRNA-96 suppresses KRAS and functions as a tumor suppressor gene in pancreatic cancer. Cancer Res 70: 6015-6025, 2010.

38. Wang G, Mao W, Zheng S and Ye J: Epidermal growth factor receptor-regulated miR-125a-5p - a metastatic inhibitor of lung cancer. FEBS J 276: 5571-5578, 2009.

39. Jiang L, Huang Q, Chang J, Wang E and Qiu X: MicroRNA HSA-miR-125a-5p induces apoptosis by activating p53 in lung cancer cells. Exp Lung Res 37: 387-398, 2011.

40. Weiss GJ, Bemis LT, Nakajima E, et al: EGFR regulation by microRNA in lung cancer: correlation with clinical response and survival to gefitinib and EGFR expression in cell lines. Ann Oncol 19: 1053-1059, 2008.

41. Cho WCS, Chow ASC and Au JSK: Restoration of tumour suppressor hsa-miR-145 inhibits cancer cell growth in lung adenocarcinoma patients with epidermal growth factor receptor mutation. Eur J Cancer 45: 2197-2206, 2009.

42. Chen Z, Zeng H, Guo Y, et al: miRNA-145 inhibits non-small cell lung cancer cell proliferation by targeting c-Myc. J Exp Clin Cancer Res 29: 151, 2010.

43. Yin R, Zhang S, Wu Y, et al: microRNA-145 suppresses lung adenocarcinoma-initiating cell proliferation by targeting OCT4. Oncol Rep 25: 1747-1754, 2011.

44. Cho WCS, Chow ASC and Au JSK: MiR-145 inhibits cell proliferation of human lung adenocarcinoma by targeting EGFR and NUDT1. RNA Biol 8: 125-131, 2011.

45. Sachdeva M and Mo YY: MicroRNA-145 suppresses cell invasion and metastasis by directly targeting mucin 1 . Cancer Res 70: 378-387, 2010.

46. Yang H, Zhang H, Zhu L, Zhang C and Li D: Identification and characterization of microRNAs in macaca fascicularis by EST analysis. Comp Funct Genomics 2012: 957607, 2012

47. van Nimwegen E: Scaling laws in the functional content of genomes. Trends Genet 19: 479-484, 2003.

48. Kim JS, Lee Y, Lee MY, et al: Multiple reaction monitoring of multiple low-abundance transcription factors in whole lung cancer cell lysates. J Proteome Res 12: 2582-2596, 2013.

49. Engelman JA, Zejnullahu K, Mitsudomi T, et al: MET amplification leads to gefitinib resistance in lung cancer by activating ERBB3 signaling. Science 316: 1039-1043, 2007.

50. Carpenter CL and Cantley LC: Phosphoinositide kinases. Curr Opin Cell Biol 8: 153-158, 1996.

51. Hennessy BT, Smith DL, Ram PT, Lu Y and Mills GB: Exploiting the PI3K/AKT pathway for cancer drug discovery. Nat Rev Drug Discov 4: 988-1004, 2005.

52. Cuevas BD, Winter-Vann AM, Johnson NL and Johnson GL: MEKK1 controls matrix degradation and tumor cell dissemination during metastasis of polyoma middle-T driven mammary cancer. Oncogene 25: 4998-5010, 2006. 
53. Hynes NE and MacDonald G: ErbB receptors and signaling pathways in cancer. Curr Opin Cell Biol 21: 177-184, 2009.

54. Wagner EF and Nebreda AR: Signal integration by JNK and p38 MAPK pathways in cancer development. Nat Rev Cancer 9: 537-549, 2009.

55. Sebolt-Leopold JS and Herrera R: Targeting the mitogen-activated protein kinase cascade to treat cancer. Nat Rev Cancer 4: 937-947, 2004

56. Gatza ML, Watt JC and Marriott SJ: Cellular transformation by the HTLV-I Tax protein, a jack-of-all-trades. Oncogene 22 5141-5149, 2003

57. van Nimwegen MJ, Verkoeijen S, van Buren L, Burg D and van de Water B: Requirement for focal adhesion kinase in the early phase of mammary adenocarcinoma lung metastasis formation. Cancer Res 65: 4698-4706, 2005.

58. Yamaguchi $\mathrm{H}$ and Condeelis J: Regulation of the actin cytoskeleton in cancer cell migration and invasion. Biochim Biophys Acta 1773: 642-652, 2007.
59. Semba S, Iwaya K, Matsubayashi J, et al: Coexpression of actin-related protein 2 and Wiskott-Aldrich syndrome family verproline-homologous protein 2 in adenocarcinoma of the lung. Clin Cancer Res 12: 2449-2454, 2006.

60. Dasgupta P, Kinkade R, Joshi B, Decook C, Haura E and Chellappan S: Nicotine inhibits apoptosis induced by chemotherapeutic drugs by up-regulating XIAP and survivin. Proc Natl Acad Sci USA 103: 6332-6337, 2006.

61. Zhang S, Togo S, Minakata K, Gu T, Ohashi R, Tajima K, Murakami A, Iwakami S, Zhang J, Xie C and Takahashi K: Distinct roles of cholinergic receptors in small cell lung cancer cells. Anticancer Res 30: 97-106, 2010.

62. Burger AM and Seth AK: The ubiquitin-mediated protein degradation pathway in cancer: therapeutic implications. Eur J Cancer 40: 2217-2229, 2004. 